\title{
State Estimation of Active Distribution Networks: Comparison Between WLS and Iterated Kalman-Filter Algorithm Integrating PMUs
}

\author{
S. Sarri, Student Member, IEEE, M. Paolone, Senior Member, IEEE, R. Cherkaoui, Senior Member, \\ IEEE, A. Borghetti, Senior Member, IEEE, F. Napolitano, Member, IEEE, C.A. Nucci, Fellow, IEEE
}

\begin{abstract}
One of the challenging tasks related to the realtime control of Active Distribution Networks (ADNs) is represented by the development of fast (i.e. sub-second) state estimation (SE) processes. As known, the problem of SE of power networks links the measurements performed in the network with a set of non-linear equations representing the links between the network node voltage phasors (i.e. the system states) and measured quantities. The calculation of these voltages is accomplished by the solution of a minimization problem by using, for instance, Weighted Least Squares (WLS) or Kalman filter (KF) methods. The availability of phasor measurement units (PMUs), characterized by high accuracy and able to directly measure node voltage phasors, allows, in principle, a simplification of the SE problem. Within this framework, the paper has two aims. The first is to propose a procedure based on the use of the Iterated KF (IKF) aiming at making achievable, in a straightforward manner, the SE of ADNs integrating PMU measurements. The second goal is to present a sensitivity analysis of the performances of WLS vs IKF methods as a function of the measurements and process covariance matrices.
\end{abstract}

Index Terms-Active distribution networks (ADNs), Kalman filter (KF), iterated Kalman filter (IKF), phasor measurement units (PMUs), state estimation (SE), smart grids.

\section{INTRODUCTION}

$\mathrm{T}$ HE increasing penetration of distributed energy resources in power distribution networks will involve a substantial evolution in the operational practice of this part of the power network infrastructure (i.e., [1], [2]). The above issues will certainly call for a massive use of advanced and smarter monitoring tools capable of increasing, in principle, the operation reliability of these networks.

In the last decade, the large use of the so-called Phasor Measurement Units (PMUs) for the monitoring of the transmission networks, has influenced the operational practices of these systems concerning the following aspects: i) system stability; ii) state estimation (SE); iii) system reliability; and iv) protections schemes (e.g. [3]-[9]). In view of the evolution of distribution networks from passive

S. Sarri, R. Cherkaoui and M. Paolone are with the École Polytechnique Fédérale de Lausanne EPFL, Lausanne, Switzerland, (e-mail: styliani.sarri@epfl.ch,mario.paolone@epfl.ch, rachid.cherkaoui@epfl.ch). to active systems, it is expected that in the future the use of PMUs will also facilitate improved operational practices of these networks.

Within the above context, one of the main challenging tasks in the operation of Active Distribution Networks (ADNs) is represented by their SE (e.g. [10]-[12]) in view of the fact that the inclusion of data gathered by PMUs certainly allows to simplify and improve the SE of ADNs (e.g. [12], [13]) $)^{1}$.

As known, classic methods used for the solution of the SE problem are based on the use of Weighted Least Squares (WLS) method (e.g. [5]). More recently, methods based on the use of the Kalman filter (KF) [15] have been proposed too. In particular, Blood et al. [16] evaluated three different algorithms in order to study the performance of a pseudodynamic system, whereas in [17] they combined the basic power flow equations with the load forecast to create a discrete-time dynamic model for SE. Gelagaev [18] made a comparison between the WLS and the Extended Kalman Filter (EKF) methods and proposed a first analysis of the relative importance of process and measurement covariance matrices. Methods used for dynamic SE have been also proposed in [19]-[25]. A further version of the KF is represented by the Iterated Kalman Filter (IKF) which is characterized by an iterative application of the EKF for the case of non-linear processes (e.g. [26] -[28]).

Within this context, the paper has two aims. The first is to propose a procedure based on the use of the IKF aiming at making achievable, in a straightforward manner, the SE of ADNs integrating PMUs. The second one is to discuss the sensitivity of the WLS vs the KF performances as a function of the measurements and process covariance matrices. This analysis aims at showing that the behaviors of these two methods are largely dependent on the selection of these matrices and, therefore, on the availability of specific measurement systems.

The structure of the paper is the following. Section II illustrates the theoretical background as well as the analytical aspects related to the proposed IKF-based SE

A. Borghetti, F. Napolitano and C.A. Nucci are with the University of Bologna, Italy, (e-mail: alberto.borghetti@unibo.it, fabio.napolitano@unibo.it, carloalberto.nucci@unibo.it).

${ }^{1}$ As discussed in [14], it is technically possible to develop specific PMUs for ADN applications by making use of hardware architectures used in standard control/monitoring environments. 
algorithm including PMU measurements. Section III presents an application example with the relevant WLS vs IKF sensitivity analysis. Section IV concludes the paper with some final remarks about the obtained results and future developments.

\section{INTEGRATING PMU DATA IN IKF STATE ESTIMATION}

This section aims at formalizing the analytical aspects related to inclusion of PMU measurements into IKF-based power systems SE.

As known, in general $\mathrm{KF}$ is a method aiming at formulating the SE problem of a general system defined by a set of independent variables $\mathbf{x} \in \mathfrak{R}^{n}$ (where $\mathfrak{R}^{n}$ is the $n-$ dimensional field of real numbers) whose discrete timeevolution is described by the following set of stochastic equations:

$$
\mathbf{x}_{k}=\mathbf{A} \mathbf{x}_{k-1}+\mathbf{B} \mathbf{u}_{k-1}+\mathbf{w}_{k-1}
$$

where:

- $\quad \mathbf{x}_{k}$ and $\mathbf{x}_{k-1}$ represent the state of the system in correspondence of discrete time steps $k$ and $k-1$ respectively;

- $\quad \mathbf{u}_{k-1} \in \mathfrak{R}^{h}$ represents a set of $h$ control variables of the system at time step $k-1$;

- $\mathbf{w}_{k-1}$ represents the system process noise assumed white and with a normal probability distribution (clearly, w $\in \mathfrak{R}^{n}$ );

- $\quad$ A is a $n \times n$ matrix that links that state of the system at time step $k-1$ with the one of the current time step $k$ for the case of null active injections and process noise;

- B is a $n \times h$ matrix that links the time evolution of the state of the system with the $h$ injections at current time step $k-1$ for the case of null process noise.

The SE is based on the availability of a set of measurements $\mathbf{z} \in \mathfrak{R}^{m}$ that are, in general, non-linearly linked with the system state by means of the following set of non-linear equations ${ }^{2}$ :

$$
\mathbf{z}_{k}=h\left(\mathbf{x}_{k}\right)+\mathbf{v}_{k}
$$

where:

- $\mathbf{z}_{k}$ represents the set of the available measurements in correspondence of the current time step $k$;

- $\quad h\left(\mathbf{x}_{k}\right)$ is a measurement function that links the set of measurements at time $k$ with the system state, at the same time step $k$, for the case of null measurement noise $\left(h\left(\mathbf{x}_{k}\right) \in \mathfrak{R}^{m}\right.$ as $\mathbf{z}_{k}$ does $)$;

- $\quad \mathbf{v}_{k}$ represents the measurement noise at the same time step $k$; it is assumed white and with a normal probability distribution. $\quad \mathbf{v}_{k}$ is also assumed

\footnotetext{
${ }^{2}$ The link between the system state, $\mathbf{x}_{k}$, and the available measurements, $\mathbf{z}_{k}$, provided by (2) could appear in the literature as a linearized set of
}

independent from $\mathbf{w}_{k}$ (clearly, $\mathbf{v}_{k} \in \mathfrak{R}^{m}$ as $\mathbf{z}_{k}$ does).

Equations (1) and (2) are those adopted for the general formulation of the KF problem [15], [29].

As known, the state of an electrical power network is associated to the identification of the phase-to-ground voltage phasors in correspondence of the $N$ network buses (e.g. [5]). Assuming to define the first bus of the network as the slack one $\left(\delta^{1}=0\right)$, the state vector $\mathbf{x}$ is composed by $n=2 N-1$ unknowns being the angles of the other network buses expressed as a function of the angle of the slack bus ${ }^{3}$ :

$$
\mathbf{x}=\left[\delta^{*, 2}, \ldots, \delta^{*, N}, V^{1}, \ldots, V^{N}\right]^{T}
$$

where $\delta^{*, i}(i=2, \ldots N)$ represents the relative phase of the voltage phasor of $i^{\text {th }}$ bus as a function of the angle of the slack bus $\delta^{1}\left(\delta^{*, i}=\delta^{i}-\delta^{1}=\delta^{i}\right)$. As discussed in [30], it is worth noting that when synchrophasors measurements are available, the selection of the bus voltage phasor angle reference in the SE problem can be suitably modified compared to the traditional approach here used. It is also important to mention that, if PMU measurements are available, at least one of these should be located in correspondence of the slack bus in order to properly define equation (3).

We can express the power injections $\mathbf{u}$ as

$$
\mathbf{u}=\left[P^{1}, \ldots, P^{N}, Q^{1}, \ldots, Q^{N}\right]^{T}
$$

where $P^{i}$ and $Q^{i} \quad(i=1, \ldots N)$ represent the active and reactive power injections (produced by generation systems, absorbed by loads or exchanged by storage systems) in correspondence of the $i^{\text {th }}$ network bus.

Let now present a form of (1) applicable to the specific case of power networks. In particular, let make use of the known formulation of the load flow problem in the implicit form.

$$
\begin{aligned}
& f_{P}^{i}(\mathbf{x}, \mathbf{u})=P^{i}-V^{i} \sum_{h=1}^{N} V^{h}\left[G^{i h} \cos \left(\delta^{i}-\delta^{h}\right)+\right. \\
& \left.+B^{i h} \sin \left(\delta^{i}-\delta^{h}\right)\right]=0 \\
& f_{Q}^{i}(\mathbf{x}, \mathbf{u})=Q^{i}-V^{i} \sum_{h=1}^{N} V^{h}\left[G^{i h} \sin \left(\delta^{i}-\delta^{h}\right)+\right. \\
& \left.-B^{i h} \cos \left(\delta^{i}-\delta^{h}\right)\right]=0
\end{aligned}
$$

By linearizing (5) as a function of the system state $\mathbf{x}$ and power injections $\mathbf{u}$ and considering that $f_{p}^{i}(\mathbf{x}, \mathbf{u})$ and $f_{Q}^{i}(\mathbf{x}, \mathbf{u})$ are implicit functions, we obtain:

equations. We have preferred to state the problem in this way as we are making use of the IKF.

${ }^{3}$ It is worth mentioning that, although distribution networks are characterized by unbalanced lines and unsymmetrical loads with consequent presence of different sequence components into the three-phase voltage and current phasors, we here consider, as a first approximation, the case of balanced networks. Therefore, we shall make reference to the direct sequence only. Moreover, the topology of the network is assumed known. 


$$
\frac{\partial \mathbf{f}(\mathbf{x}, \mathbf{u})}{\partial \mathbf{x}} \Delta \mathbf{x}+\frac{\partial \mathbf{f}(\mathbf{x}, \mathbf{u})}{\partial \mathbf{u}} \Delta \mathbf{u}+\mathbf{e}=0
$$

where

$$
\mathbf{f}(\mathbf{x}, \mathbf{u})=\left[\mathbf{f}_{P}(\mathbf{x}, \mathbf{u}), \mathbf{f}_{Q}(\mathbf{x}, \mathbf{u})\right]
$$

Equation (6) can be also written as:

$$
\overbrace{\left[\begin{array}{cc}
-\frac{\partial \mathbf{f}_{P}(\mathbf{x}, \mathbf{u})}{\partial \delta} & -\frac{\partial \mathbf{f}_{P}(\mathbf{x}, \mathbf{u})}{\partial V} \\
-\frac{\partial \mathbf{f}_{Q}(\mathbf{x}, \mathbf{u})}{\partial \delta} & -\frac{\partial \mathbf{f}_{Q}(\mathbf{x}, \mathbf{u})}{\partial V}
\end{array}\right]}^{J} \Delta \mathbf{x}+\overbrace{\left[\begin{array}{cc}
I & 0 \\
0 & I
\end{array}\right]}^{I} \Delta \mathbf{u}+\mathbf{e}=0
$$

Equations (6) or (8) appear closer to (1). Indeed, we can rewrite (8) as follows:

$$
\mathbf{x}_{k}=\mathbf{x}_{k-1}+\mathbf{J}_{k}^{-1}\left(\mathbf{u}_{k}-\mathbf{u}_{k-1}\right)+\mathbf{J}_{k}^{-1} \mathbf{e}
$$

The term $\mathbf{J}_{k}^{-1} \mathbf{e}$ of (9) corresponds to the system process noise $\mathbf{w}_{k}$ of (1) and, in our case, is due to the error of the linearization process of (5).

Equation (9) may be adopted as a pseudo-dynamic model of the network with matrix $\mathbf{A}$ of (1) equal to the identity matrix. However, in KF-based SE methods this equation is only used to provide the so-called a-priori estimation of the system state at a given time step. In principle, the proximity of the a-priori state predicted by (9) to the real one has a direct influence on the number of iterations that the KF has to perform to converge. As this aspect deserves specific investigations, we are here assuming for the a-priori estimated state the so-called 'flat-start' composed by voltage angles are equal to zero and voltage magnitudes equal to one in per-unit:

$$
\mathbf{x}_{k, 0}=[\overbrace{0, \ldots, 0,1, \ldots, 1}^{N-1}]^{N}
$$

where $\mathbf{x}_{k, 0}$ indicates the a-priori state in correspondence of the generic $k$-th time step ${ }^{4}$.

After the definition of $\mathbf{x}_{k, 0}$, the IKF algorithm starts an iterative process that, at the generic time step $k$, aims at minimizing the a-posteriori error by means of a non-linear combination of an a-priori state estimate and a weighted difference between the current measurement $\mathbf{z}_{k}$ and a measurement prediction $h\left(\mathbf{x}_{k, i}\right)$ [29].

$$
\mathbf{x}_{k, i+1}=\mathbf{x}_{k, i}+\mathbf{K}_{k, i}\left[\mathbf{z}_{k}-h\left(\mathbf{x}_{k, i}\right)\right]
$$

where $\mathbf{K}$ is the so-called KF blending factor or Kalman gain. In what follows we will define in detail all the

\footnotetext{
${ }^{4}$ As it will be clarified next, subscript ' 0 ' of (12) corresponds to the KF process is at iteration zero.
}

elements appearing in (11).

The term

$$
\boldsymbol{\gamma}_{k}=\mathbf{z}_{k}-h\left(\mathbf{x}_{k, i}\right)
$$

is called measurement innovation (e.g. [29], [31]) and it represents the new information brought by the latest observation.

Now, we should express (2), or, in other terms (12), for the problem of interest. In order to include the PMU data in our algorithm, the set of measurements is assumed to be composed as follows:

- type- $d$ nodes where we assume to measure phase-toground voltage phasors by means of PMU devices;

- type- $s$ nodes where we assume to measure active and reactive power injections so that $d+s \geq N$.

The relevant array $\mathbf{z}$ of measurements defined by (2) is, therefore, the following:

$$
\mathbf{z}=[\overbrace{\delta^{2}, \ldots, \delta^{d}, V^{1}, \ldots, V^{d}}^{z^{d}}, \overbrace{P^{1}, \ldots, P^{s}, Q^{1}, \ldots, Q^{s}}^{z^{s}}]^{T}
$$

As the definition of the blending factor, or Kalman gain $\mathbf{K}$, is based on a linearized link between the system state $\mathbf{x}$ and the measurements $\mathbf{z}$, we need to derive a linearized version of (2) which can be written as follows:

$$
\mathbf{z}_{k}=\mathbf{H}_{k} \mathbf{x}_{k}+\mathbf{v}_{k}^{\prime}
$$

where $\mathbf{H}_{k}$ represents the above-cited linear dependency and $\mathbf{v}^{\prime}{ }_{k}$ a combined measurement and $h\left(\mathbf{x}_{k}\right)$-linearization errors.

Let define a $(2 d-1) \times(2 N-1)$ matrix $\mathbf{T}$ composed by the rows of the $(2 N-1)$ identity matrix corresponding to the type- $d$ nodes where phase-to-ground voltage phasors are measured by PMUs. Matrix $\mathbf{T}$ allows linking the first part of the measurement array $\mathbf{z}_{k}^{d}$ to the system state variables.

$$
\mathbf{z}_{k}^{d}=\mathbf{T} \mathbf{x}_{k}+\mathbf{v}_{k}^{d}
$$

where, the term $\mathbf{v}_{k}^{d}$ represents the array of the uncertainties of the state variables measured by the PMUs.

We should now find a linear expression that allows to link the measured injected power into type- $s$ nodes, namely $\mathbf{z}_{k}^{s}$, to the state of the system. For this purpose, we can use (8):

$$
\begin{aligned}
& {\left[\begin{array}{c}
-\frac{\partial \mathbf{f}_{P}^{s}(\mathbf{x}, \mathbf{u})}{\partial \delta}-\frac{\partial \mathbf{f}_{P}^{s}(\mathbf{x}, \mathbf{u})}{\partial V} \\
-\frac{\partial \mathbf{f}_{Q}^{s}(\mathbf{x}, \mathbf{u})}{\partial \delta}-\frac{\partial \mathbf{f}_{Q}^{s}(\mathbf{x}, \mathbf{u})}{\partial V}
\end{array}\right]\left(\mathbf{x}_{k}-\mathbf{x}_{k-1}\right)+} \\
& +\left(\mathbf{z}_{k}^{s}-\mathbf{z}_{k-1}^{s}\right)+\mathbf{e}=0
\end{aligned}
$$


where terms $\frac{\partial \cdot^{s}}{\partial \cdot}$ refer to the rows of the Jacobian matrix $\mathbf{J}$ relevant to type- $s$ nodes and $\mathbf{z}_{k}^{s}, \mathbf{z}_{k-1}^{s}$ to the measured injected active and reactive powers in correspondence to type $-s$ nodes.

From (16), we can then infer $\mathbf{z}_{k}^{s}$ :

$$
\mathbf{z}_{k}^{s}=\left[\begin{array}{ll}
\frac{\partial \mathbf{f}_{P}^{s}(\mathbf{x}, \mathbf{u})}{\partial \delta} & \frac{\partial \mathbf{f}_{P}^{s}(\mathbf{x}, \mathbf{u})}{\partial V} \\
\frac{\partial \mathbf{f}_{Q}^{s}(\mathbf{x}, \mathbf{u})}{\partial \delta} & \frac{\partial \mathbf{f}_{Q}^{s}(\mathbf{x}, \mathbf{u})}{\partial V}
\end{array}\right] \mathbf{x}_{k}+\mathbf{v}_{k}^{s}
$$

where the term $\mathbf{v}_{k}^{s}$ is given by:

$$
\mathbf{v}_{k}^{s}=-\left[\begin{array}{ll}
\frac{\partial \mathbf{f}_{P}^{s}(\mathbf{x}, \mathbf{u})}{\partial \delta} & \frac{\partial \mathbf{f}_{P}^{s}(\mathbf{x}, \mathbf{u})}{\partial V} \\
\frac{\partial \mathbf{f}_{Q}^{s}(\mathbf{x}, \mathbf{u})}{\partial \delta} & \frac{\partial \mathbf{f}_{Q}^{s}(\mathbf{x}, \mathbf{u})}{\partial V}
\end{array}\right] \mathbf{x}_{k-1}^{s}+\mathbf{z}_{k-1}^{s}-\mathbf{e}
$$

The term $\mathbf{v}_{k}^{s}$ is clearly not constant with the time step $k$ and represents an equivalent uncertainty that combines the one of the type- $s$ measurements, with the one related to the approximation of (8).

Therefore, (2) could be expressed as follows:

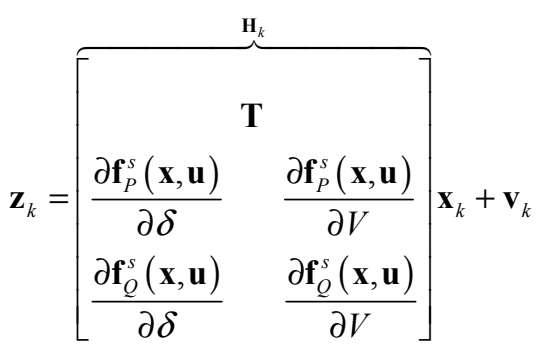

where

$$
\mathbf{v}_{k}=\left[\begin{array}{c}
\mathbf{v}_{k}^{d} \\
\mathbf{v}_{k}^{s}
\end{array}\right]
$$

The only matrix that remains to be defined is the blending factor K. As defined in [29], [32], and [33], the typical form to express this matrix in order to make (11) a converging process is the following.

$$
\mathbf{K}_{k, i}=\mathbf{P}_{k, i-1} \mathbf{H}_{k}^{T}\left(\mathbf{H}_{k} \mathbf{P}_{k, i-1} \mathbf{H}_{k}^{T}+\mathbf{R}_{k}\right)^{-1}
$$

where $\mathbf{R}_{k}$ is the diagonal matrix associated to the uncertainties of the measurements:

$$
\mathbf{R}_{k}=\left[\begin{array}{ccc}
v_{k, 11} & \cdots & 0 \\
\vdots & \ddots & \vdots \\
0 & \cdots & v_{k, m m}
\end{array}\right]
$$

where $v_{k, j j}$ are the elements of the array $\mathbf{v}_{k}$, and $\mathbf{P}_{k, i-1}$ of (21) is the a-priori estimate covariance matrix defined as:

$$
\mathbf{P}_{k, i-1}=\mathbf{A} \mathbf{P}_{k-1} \mathbf{A}^{T}+\mathbf{Q}_{k-1}
$$

In our case, matrix $\mathbf{A}$ is the identity matrix $\mathbf{I}$ and $\mathbf{Q}_{k-1}$ represents the process covariance matrix, namely the approximations introduced into the linearization of the process. It is worth observing that in the literature the value of matrix $\mathbf{Q}_{k-1}$ is usually selected arbitrarily although, in principle, it could be computed if the process is known.

As the relative values of $\mathbf{Q}_{k-1}$ and $\mathbf{R}_{k}$ directly influence the behavior of the IKF, they will be the subject of the sensitivity analysis reported in the next section. In our case we assume the values of $\mathbf{Q}_{k-1}$ known and constant for different time steps $k$ (i.e. $\mathbf{Q}_{k-1}=\mathbf{Q}$ ).

The iterative process is, then, the following.

i) Iteration $i=0$ :

- construction of the state $\mathbf{x}_{k, 0}$ by using (10);

- projection of the error covariance ahead:

$$
\mathbf{P}_{k, 0}=\mathbf{P}_{k-1}+\mathbf{Q}
$$

ii) Iteration $i>0$ :

- Computation of the blending factor by using (21);

- update of the state:

$$
\mathbf{x}_{k, i}=\mathbf{x}_{k, i-1}+\mathbf{K}_{k, i}\left[\mathbf{z}_{k}-\mathbf{h}_{k}\left(\mathbf{x}_{k, i-1}\right)\right]
$$

- update of the error covariance matrix:

$$
\mathbf{P}_{k, i}=\left(\mathbf{I}-\mathbf{K}_{k, i} \mathbf{H}_{k}\right) \mathbf{P}_{k, i-1}
$$

The stopping criterion applies to the absolute values of differences between two consecutive iterations of phase-toground voltages angles and magnitudes. In particular, the following criteria have to be satisfied simultaneously. The values of $\varepsilon_{\delta}$ and $\varepsilon_{V}$ are suitably chosen as a function of the values of phase-to-ground voltages angles and magnitudes.

$$
\begin{aligned}
& \max \left(\left|\boldsymbol{\delta}_{k, i}-\boldsymbol{\delta}_{k, i-1}\right|\right)<\varepsilon_{\delta} \\
& \max \left(\left.|| \overline{\mathbf{V}}\right|_{k, i}-|\overline{\mathbf{V}}|_{k, i-1} \mid\right)<\varepsilon_{V}
\end{aligned}
$$

\section{AppliCATION EXAMPLE AND SENSITIVITY ANALYSIS}

Fig. 1 shows the IEEE 13-bus distribution test feeder [34] that has been used to obtain the results reported in this section. Bus 1 has been assumed to be the slack of the system as it represents the connection to the subtransmission network. The test feeder has been considered to be balanced, therefore, as mentioned in note 1 of Section II, we have made reference to the direct sequence only. 


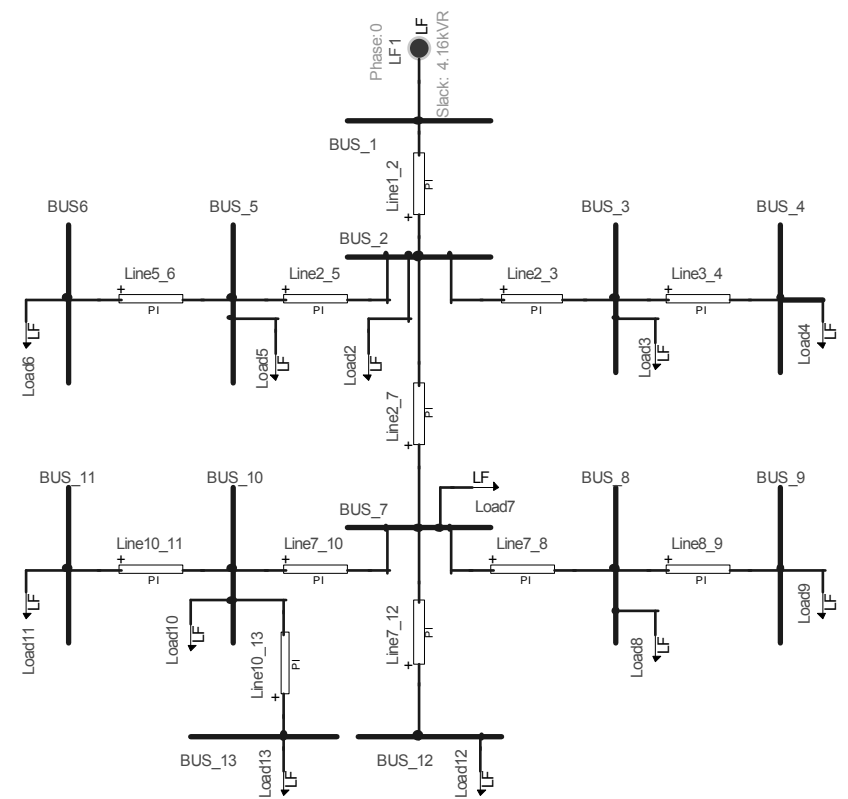

Fig. 1. The simulated IEEE 13-bus distribution test feeder [34].

The configuration of the measurement points, namely, measured phasors and active-reactive power injections, is reported in Table I. The daily total active and reactive load profiles are shown in Fig. 2. They are separated by 15 minutes time intervals. The loads are not equally distributed in the system and their repartition is a function of a-priori assumed rated power of secondary substations.

TABLE I

TyPe OF MEASUREMENTS IN THE SimULATED NETWORK

\begin{tabular}{ccc}
\hline \hline $\begin{array}{c}\text { Bus } \\
\text { Number }\end{array}$ & PMU measurements & PQ measurements \\
\hline 1 & $\checkmark$ & $\checkmark$ \\
\hline 2 & & $\checkmark$ \\
\hline 3 & $\checkmark$ & $\checkmark$ \\
\hline 4 & & $\checkmark$ \\
\hline 5 & $\checkmark$ & $\checkmark$ \\
\hline 6 & & $\checkmark$ \\
\hline 7 & $\checkmark$ & $\checkmark$ \\
\hline 8 & $\checkmark$ & $\checkmark$ \\
\hline 9 & & $\checkmark$ \\
\hline 10 & $\checkmark$ & $\checkmark$ \\
\hline 11 & & $\checkmark$ \\
\hline 12 & & $\checkmark$ \\
\hline 13 & & $\checkmark$ \\
\hline \hline
\end{tabular}

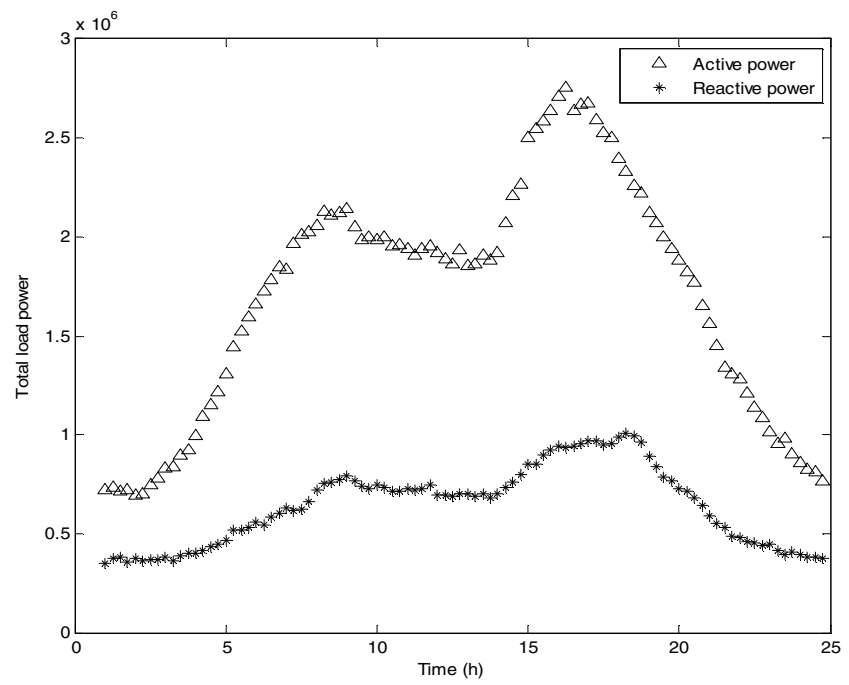

Fig. 2. Adopted daily total active and reactive load profiles.
The procedure adopted to evaluate the accuracy of the state estimation is described below.

1) For each time step (i.e. every $15 \mathrm{~min}$ ), a load flow is computed to determine the true state of the system.

2) The measured quantities are provided by modifying their true values obtained from the previous step 1) and adding randomly-generated errors which are function of assumed standard deviations of the measurements error distributions.

3) On the basis of the set of measurements provided by the previous step 2), the estimation of the system state is achieved by using two different computational methods, namely the IKF algorithm described in Section II and the WLS (e.g. [5]).

The comparison between the two algorithms is performed for different measurements ( $\mathbf{R})$ and process covariance ( Q ) matrices [29]. Such an analysis is of importance as these two quantities are largely influencing the performances of the $\mathrm{KF}$ process. In particular, $\mathbf{R}$ is weighting how much the KF trusts the measurements, whereas $\mathbf{Q}$ weights how much the KF trusts the estimated values. Tuning the SE-KF involves achieving suitable values for $\mathbf{R}$ and $\mathbf{Q}$ matrices, so that the best estimation accuracy is obtained [35]. For general matrices we need to compare the eigenvalues of $\mathbf{Q}$ and $\mathbf{R}$. In case $\mathbf{Q}$ and $\mathbf{R}$ are diagonal with all equal terms say $Q$ and $R$ ( $\mathbf{Q}=Q \mathbf{I}, \mathbf{R}=R \mathbf{I}$ ), we can define a 'tuning ratio' $Q / R$. As $R$ gets larger and $Q$ gets smaller, the tuning ratio becomes smaller, whereas as $R$ gets smaller and $Q$ gets larger, it becomes larger. Therefore, two different cases have been analyzed for each of the above-mentioned conditions. Table II reports the specific values adopted for $R$ and $Q$ concerning Cases I and II. With reference to Case I, Fig. 3 shows the true and estimated magnitudes and phases of direct-sequence voltage in correspondence of bus 7 of Fig. 1 obtained by using WLS and IKF methods. As in this case the tuning ratio is small, the IKF estimations are characterized by a lower quality, as expected, compared to those provided by WLS. On the other hand, Fig. 4 shows the comparative performance of the two methods for a larger value of the tuning ratio (see Table II). In this case, the IKF gives results characterized by the same level of accuracy as WLS. These results might be influenced by the type and location of available measurements and will be the subject of further investigations.

TABLE II

Selected Values of the Measurements ( $\mathbf{R}$ ) and Process ( $\mathbf{Q}$ ) COVARIANCE MATRICES FOR Fig. 3 AND 4

\begin{tabular}{|c|c|c|c|}
\hline \multicolumn{2}{|r|}{ Quantity } & Case I & Case II \\
\hline \multirow{4}{*}{$R$} & \multirow{4}{*}{$\begin{array}{c}\text { PMU RMS variance [p.u.] } \\
\text { PMU phase variance [rad] } \\
\text { Active power variance [p.u.] } \\
\text { Reactive power variance [p.u.] }\end{array}$} & $5.778 \cdot 10^{-5}$ & $5.778 \cdot 10^{-4}$ \\
\hline & & $6.561 \cdot 10^{-5}$ & $6.561 \cdot 10^{-4}$ \\
\hline & & $4.000 \cdot 10^{-5}$ & $4.000 \cdot 10^{-4}$ \\
\hline & & $4.000 \cdot 10^{-5}$ & $4.000 \cdot 10^{-4}$ \\
\hline & $Q$ & $10^{-7}$ & $10^{-1}$ \\
\hline & Tuning ratio $Q / R$ & $10^{-2}$ & $10^{3}$ \\
\hline
\end{tabular}



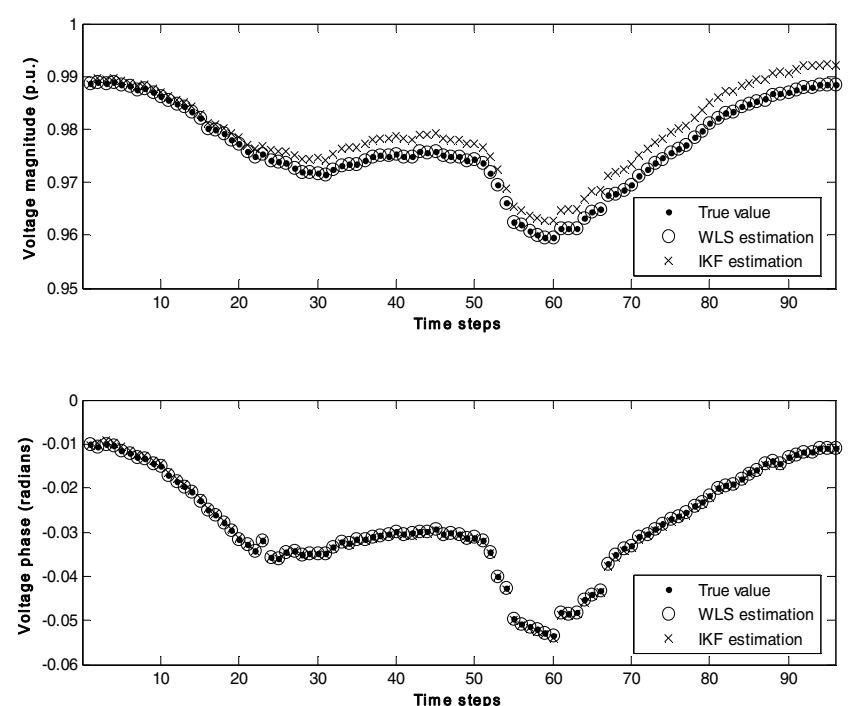

a)
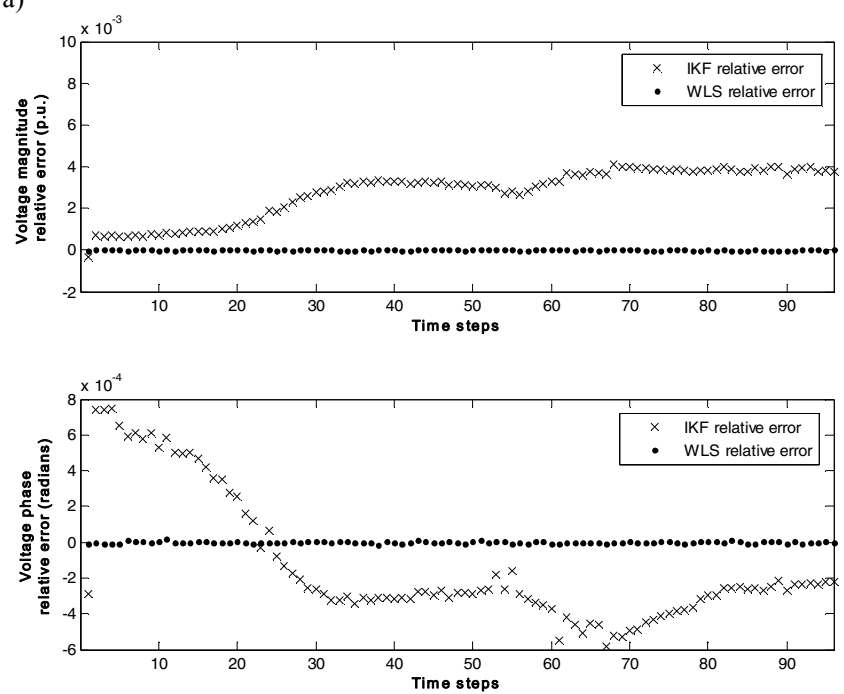

Fig. 3. True and estimated values of the direct-sequence voltage phasor in correspondence of node 7 of the IEEE 13 nodes test distribution feeder of Fig. 1 for values of $R$ and $Q$ corresponding to Case I of Table II: a) absolute magnitude and phase of voltage phasor; b) relative errors of magnitude and phase of voltage phasor.

TABLE III

Selected Values of the Measurements ( $\mathbf{R}_{k}$ ) And Process ( $\mathbf{Q}$ ) COVARIANCE MATRICES FOR FIG. 5

\begin{tabular}{|c|c|c|}
\hline \multicolumn{2}{|r|}{ Quantity } & Value \\
\hline \multirow{4}{*}{$R$} & \multirow{4}{*}{$\begin{array}{c}\text { PMU RMS variance [p.u.] } \\
\text { PMU phase variance [rad] } \\
\text { Active power variance [p.u.] } \\
\text { Reactive power variance [p.u.] }\end{array}$} & $5.778 \cdot 10^{-6}$ \\
\hline & & $6.561 \cdot 10^{-6}$ \\
\hline & & $4.000 \cdot 10^{-6}$ \\
\hline & & $4.000 \cdot 10^{-6}$ \\
\hline & $Q$ & {$\left[10^{-3} \div 10^{-7}\right]$} \\
\hline
\end{tabular}

TABLE IV

Selected Values of the Measurements ( $\mathbf{R}_{k}$ ) And Process ( $\mathbf{Q}$ ) COVARIANCE MATRICES FOR FIG. 6

\begin{tabular}{|c|c|c|}
\hline & Quantity & Value \\
\hline \multirow{4}{*}{$R$} & \multirow{4}{*}{$\begin{array}{c}\text { RMS var. [p.u.] } \\
\text { phase var. [rad] } \\
\text { Act. power var. [p.u.] } \\
\text { React. power var. [p.u.] }\end{array}$} & $5.778 \cdot\left[10^{-3} \div 10^{-7}\right]$ \\
\hline & & $6.561 \cdot\left[10^{-3} \div 10^{-7}\right]$ \\
\hline & & $4.000 \cdot\left[10^{-3} \div 10^{-7}\right]$ \\
\hline & & $4.000 \cdot\left[10^{-3} \div 10^{-7}\right]$ \\
\hline & $Q$ & $10^{-4}$ \\
\hline
\end{tabular}
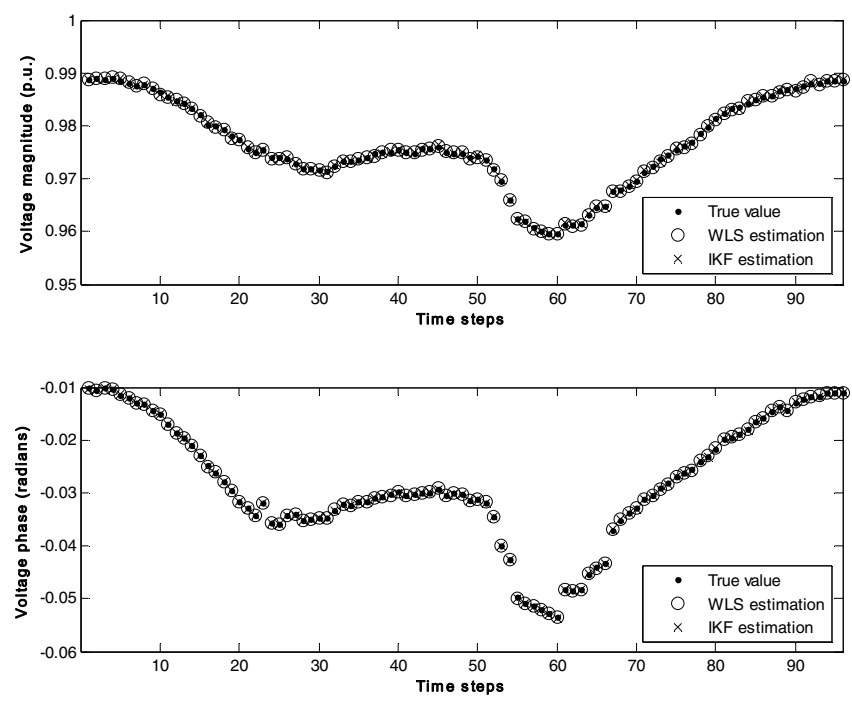

a)
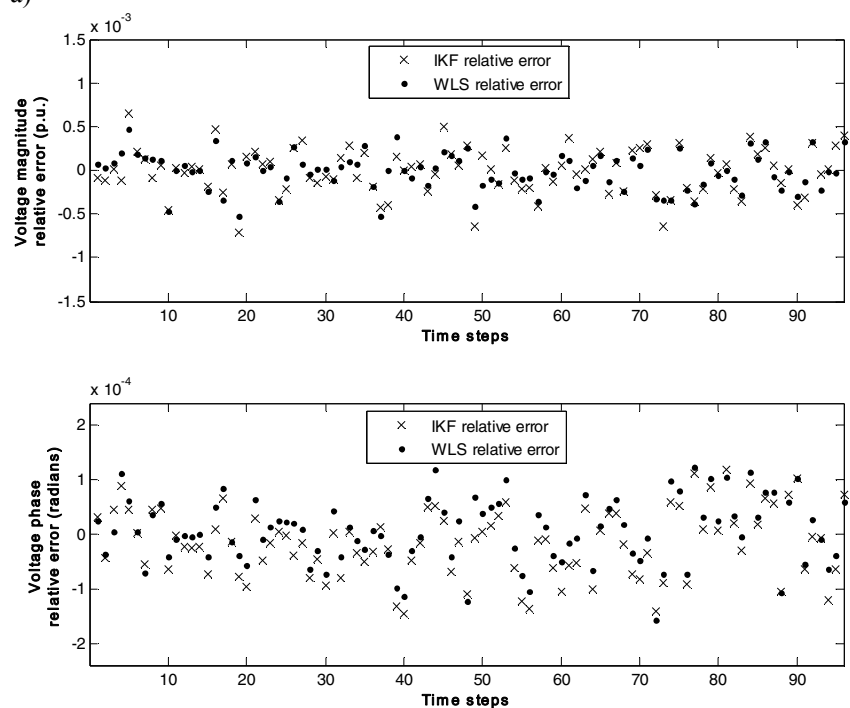

b)

Fig. 4. True and the estimated values of the direct-sequence voltage phasor in correspondence of node 7 of the IEEE 13 nodes test distribution feeder of Fig. 1 for values of $R$ and $Q$ corresponding to Case II of Table II: a) absolute magnitude and phase of voltage phasor; b) relative errors of magnitude and phase of voltage phasor.

Fig. 5 shows the norm variation of the relative errors related to all bus-voltage magnitudes and phases for a specific time step (the rest of the time steps provide similar behavior). These results are obtained for a constant value of $R$ and different values of $Q$ as reported in Table III. Fig. 6 shows the complementary results of Fig. 5 but with a constant value of $Q$ and different values of $R$, as reported in Table IV.

As it can be observed from Fig. 5 and Fig. 6, there could be a trade-off in the performance of IKF and WLS as a function of the values assumed by $\mathbf{Q}$ and $\mathbf{R}$.

This can be justified, by taking a more thorough look to (21). The following term, that is a component of the Kalman Gain $\mathbf{K}$, is called measurement prediction covariance matrix:

$$
\mathbf{S}_{k, i}=\mathbf{H}_{k} \mathbf{P}_{k, i-1} \mathbf{H}_{k}^{T}+\mathbf{R}_{k}
$$

Assuming increasing values of $\mathbf{R}_{k}$, in general, elements of matrix $\mathbf{S}$ increase, but, due to the use of the inverse $\mathbf{S}^{-1}$ 
in the definition of $\mathbf{K}$ in (21), the overall weight of $\mathbf{K}$ decreases, which leads to an overall decrease of the contribution of the measurement innovation $\gamma$ in the update equation (25). On the other hand, increasing values of $\mathbf{Q}$ and, as a consequence of $\mathbf{P}$, might lead to higher values for $\mathbf{S}$. However, it is worth noting that $\mathbf{P}$ is also adopted for the calculation of $\mathbf{K}$ where $\mathbf{S}^{-1}$ is used as follows:

$$
\mathbf{K}_{k, i}=\mathbf{P}_{k, i-1} \mathbf{H}_{k}^{T} \mathbf{S}_{k, i}{ }^{-1}
$$

In (29) compared to $\mathbf{S}$, the term $\mathbf{P}_{k, i-1}$ plays a more important role in the calculation of $\mathbf{K}$ as $\mathbf{P}_{k, i-1}$ is directly derived from $\mathbf{Q}$ (see equation (23)). Therefore, increasing values of $\mathbf{Q}$ results in increasing $\mathbf{K}$, which leads to an increased contribution of the measurement innovation $\gamma$ in the update equation (25).
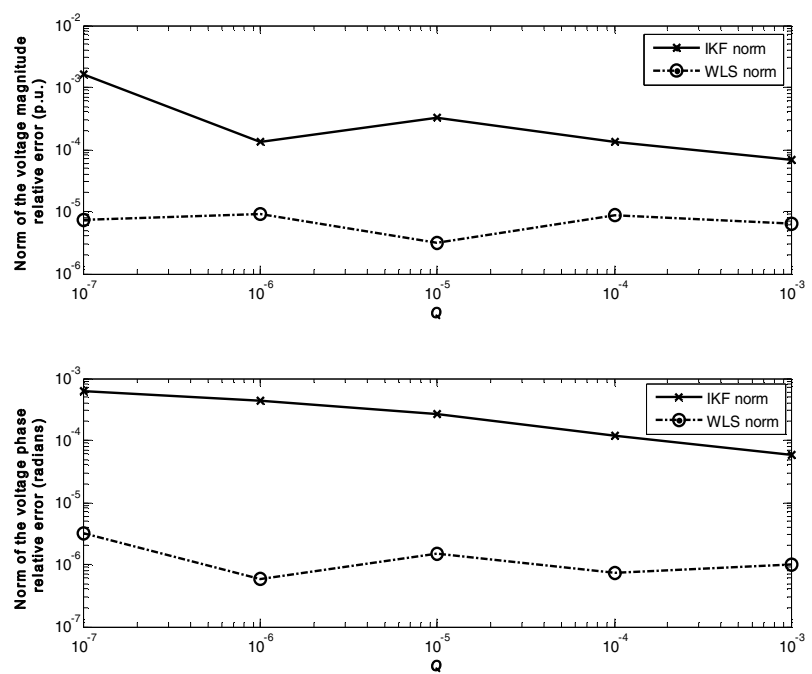

Fig. 5. Variation of the norms of the voltage phase and magnitude relative errors, for a specific time step (time step 53) and for a constant value of $R$ (shown in Table III), as a function of $Q$.
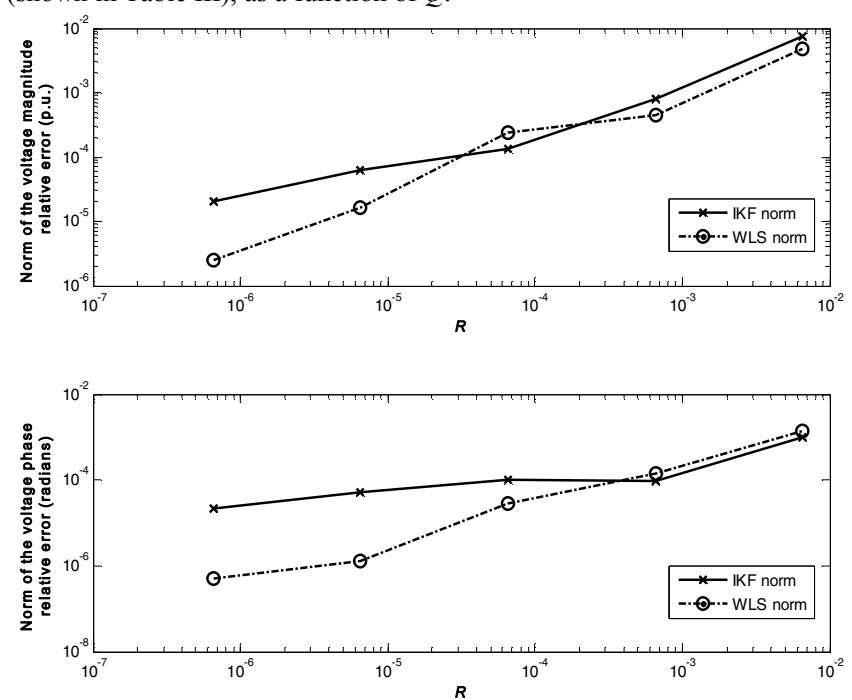

Fig. 6. Variation of the norms of the voltage phase and magnitude relative errors, for a specific time step (time step 53) and for a constant value of $Q$ (shown in Table IV), as a function of $R$.

\section{CONCLUSIONS}

The paper has shown the derivation of a SE algorithm based on the use of IKF integrating measurements coming from PMUs and nodal power injections. The algorithm has been conceived in order to specifically take advantage of PMUs measurements.

By making reference to the IEEE 13-bus test feeder, the paper has shown a comparative analysis of the developed IKF algorithm in comparison to the traditional WLS one. In particular, this analysis has been focused on the evaluation of measurements and process covariance error matrices on the performances of the two SE methods. In particular, the paper has shown that the performances of WLS and IKF SE methods are largely dependent on the process and measurement covariance matrices and that their evaluation plays an important role in the selection of the proper SE algorithm.

Further research will focus on the deterministic calculation of $\mathbf{Q}$, on the influence of type and location of available measurements and, finally, on the computational/performance improvements of the proposed IKF algorithm.

\section{REFERENCES}

[1] N. Jenkins, R. Allan, P. Crossley, D. Kirschen, and G. Strbac, Embedded Generation. $\quad$ London, U.K.: Inst. Elect. Eng., 2000.

[2] W. Allen, "Effects of wide-area control on the protection and operation of distribution networks," in Power Systems Conference, 2009, PSC 2009, Clemson, South Carolina, USA, pp. 1-10.

[3] S. E. Stanton, C. Slivinsky, K. Martin, and J. Nordstrom, "Application of phasor measurements and partial energy analysis in stabilizing large disturbances," IEEE Trans. on Power Systems, vol. 10, issue 1, pp: 297 - 306, Feb. 1995.

[4] A. G. Phadke and J. S. Thorp, Synchronized Phasor Measurements and Their Application. New York: Springer, 2008.

[5] A. Abur and A. G. Exposito, Power System State Estimation-Theory and Implementation. New York: CRC Press, M. Dekker, 2004.

[6] A. Ishibashi, M. Imai, K. Omata, S. Sato, T. Takagi, Y. Nakachi, and S. Ogawa, "New type of islanding detection system for distributed generation based on voltage angle difference between utility network and distributed generation site," in Proc. 8th IEE Int. Conf. Develop. Power Syst. Protection, 2004, vol. 2, pp. 542-545.

[7] D. M. Laverty, D. J. Morrow, R. J. Best, and P. A. Crossley, "Differential ROCOF relay for loss-of-mains protection of renewable generation using phasor measurement over internet protocol," in Proc. CIGRE/IEEE Power Energy Soc. Joint Symp. Integr. Wide-Scale Renewable Resources Power Del. Syst., Calgary, AB, Canada, Jul. 29-31, 2009, pp. 1-7.

[8] O. Samuelsson, M. Hemmingsson, A. H. Nielsen, K. O. H. Pedersen, and J. Rasmussen, "Monitoring of power system events at transmission and distribution level," IEEE Trans. Power Syst., vol. 21, no. 2, pp. 1007-1008, 2006.

[9] J. Tlusty, A. Kasembe, Z. Muller, J. Svec, T. Sykora, A. Popelka, E. V. Mgaya, and O. Diallo, "The monitoring of power system events on transmission and distribution level by the use of phasor measurements units (PMU)," in Proc. 20th Int. Conf. Exhib. Elect. Distrib. (CIRED), Prague, Czech Republic, Jun. 8-11, 2009.

[10] A. G. Exposito, A. de la Jaen, and J. L. R. Izaga, "An alternative state estimation formulation for radial distribution networks," in Proc. of the 2007 IEEE Lausanne PowerTech, 2007, pp. 396-400.

[11] M. M. Nordman, and M. Lehtonen, "Distributed agent-based state estimation for electrical distribution networks," IEEE Trans. on PWRS, vol. 20, no. 2, pp. 652-658, 2005.

[12] M. Powalko, K. Rudion, P. Komarnicki, and J. Blum-schein, "Observability of the distribution system," in Proc. 20th Int. Conf. Exhib. Elect. Distrib. (CIRED), Prague, Czech Republic, Jun. 8-11, 2009.

[13] A. P. S. Meliopoulos, G. J. Cokkinides, F. Galvan, and B. Farnadesh, "Distributed state estimator-Advances and demostration," in Proc. of the 41st Hawaii International Conference on System Sciences - 2008, Waikoloa, Hawaii, USA, Jan. 7-10, 2008.

[14] M. Paolone, A. Borghetti, and C. A. Nucci, "A synchrophasor estimation algorithm for the monitoring of active distribution networks in steady state and transient conditions," in Proc. of the $17^{\text {th }}$ Power Systems Computation Conference (PSCC 2011), Aug. 22-26, 2011, Stockholm, Sweden, vol. 1, pp. 213-220. 
[15] R. E. Kalman, "A new approach to linear filtering and prediction problems," Transaction of the ASME-Journal of Basic Engineering, pp. 33-45, 1960.

[16] E. A. Blood, M. D. Ilic, J. Ilic, and B. H. Krogh, "A Kalman filter approach to quasi-static state estimation in electric power systems," in Proc. of the 38th North American Power Symposium, 2006 (NAPS 2006), 17-19 Sept. 2006, Carbondale, IL, USA, pp. 417-422.

[17] E. A. Blood, B. H. Krogh, and M. D. Ilic, "Electric power system static state estimation through Kalman filtering and load forecasting," in Proc. of the IEEE PES General Meeting-Conversion and Delivery of Electrical Energy in the 21st Century, Pittsburgh, PA, USA, Hawaii, USA, July 20-24, 2008, pp. 1-6.

[18] R. Gelagaev, P. Vermeyen, and J. Driesen, "State estimation in distribution grids," in Proc. of the 13th International Conference on Harmonics and Quality of Power 2008, Wollongong, Australia, Sept. 28-Oct. 1, 2008, pp. 1-6.

[19] G. Durgaprasad, and S. S. Thakur, "Robust dynamic state estimation of power systems based on m-estimation and realistic modeling of system dynamics," IEEE Trans. Power Syst., vol. 13, no. 4, pp. 13311336, November 1998.

[20] A. K. Sinha, and J. K. Mondal, "Dynamic state estimator using ANN based bus load prediction," IEEE Trans. Power Syst., vol. 14, no. 4, pp. 1219-1225, November 1999.

[21] K.-R. Shih, and S.-J. Huang, "Application of a robust algorithm for dynamic state estimation of a power system," IEEE Trans. Power Syst., vol. 17, no. 1, pp. 141-147, February 2002.

[22] Z. Huang, K. Schneider, and J. Nieplocha, "Feasibility studies of applying kalman filter techniques to power system dynamic state estimation," in Proc. of the International Power Engineering Conference 2007 (IPEC 2007), Dec. 3-6, 2007, Singapore, pp. 376382.

[23] E. Ghahremani, and I. Kamwa, "Dynamic state estimation in power system by applying the extended kalman filter with unknown inputs to phasor measurements," IEEE Trans. Power Syst., vol. 26, no. 4, pp. 2556-2566, November 2011.

[24] J. A. de la O Serna, and J. R.-Maldonado, "Instantaneous oscillating phasor estimates with Taylor ${ }^{K}-$ Kalman filters," IEEE Trans. Power Syst., vol. 26, no. 4, pp. 2336-2344, November 2011.

[25] J. Zhang, G. Welch, G. Bishop, and Z. Huang, "Reduced measurement-space dynamic state estimation (ReMeDySE) for power systems," in Proc. of the 2011 IEEE Trondheim PowerTech, 2011, pp. $1-7$.

[26] A. S. Debs, and R. E. Larson, "A dynamic estimator for tracking the state of a power system," IEEE Trans. on Power Apparatus and Systems, vol. PAS-89, no. 7, pp. 1670-1678, Sept.-Oct. 1970.

[27] B.M. Bell, F.W. Cathey, "The Iterated Kalman filter update as a Gauss-Newton method", IEEE Transactions on Automatic Control, Vol. 38(2), Feb 1993.

[28] N. Xin-liang, Z. Guo-qing, L. Yuan-hua, and C. Hong, "An improvement on the iterated Kalman filter," in Proc. of the Radar Conference, 2009 IET International, Guillin, China, April 20-22, 2009, pp. 1-4.

[29] G. Welch and G. Bishop, An introduction to the Kalman Filter. TR 95-041, Dep. of Computer Science, University of North Carolina, USA, July 2006.

[30] Jun Zhu, A. Abur, "Effect of Phasor Measurements on the Choice of Reference Bus for State Estimation", Proc. of the 2007 IEEE PES General Meeting, 2007.

[31] M. I. Ribeiro, Kalman and Extended Kalman Filters: Concept, Derivation and Properties, Institute for Systems and Robotics, Instituto Superior Téenico, Lisboa, Portugal, February 2004.

[32] R. G. Brown, and P. Y. C. Hwang, Introduction to Random Signals and Applied Kalman Filtering. Second Edition, John Wiley \& Sons, Inc. 1992.

[33] O. L. R. Jacobs, Introduction to Control Theory. Second Edition, Oxford University Press, 1993.

[34] IEEE Distribution Planning Working Group, "Radial distribution test feeders," IEEE Trans. Power Syst., vol. 6, pp. 975-985, 1991.

[35] R. N. Banavar, and A. Nath, Notes on Kalman Filter, Systems \& Control Eng., Indian Institute of Technology, Bombay, India, March 2004.

\section{BIOGRAPHIES}

Styliani Sarri was born in Larisa, Greece, in 1986. In 2011 she graduated in electrical engineering from the Aristotle University of Thessaloniki (AUTh), Thessaloniki, Greece.

In 2011, she joined the Distributed Electrical System laboratory (DESL) at the École Polytechnique Fédérale de Lausanne (EPFL), Lausanne,
Switzerland, where she is pursuing the Ph.D. degree in electrical engineering. Her current research interests include distribution network monitoring and state estimation and real-time operation of electrical grids with special reference to power distribution networks.

Mario Paolone (M'07-SM'10) was born in Campobasso, Italy, in 1973 He received the M.Sc. degree (with honors) in electrical engineering and the Ph.D. degree from the University of Bologna, Italy, in 1998 and 2002, respectively.

In 2005, he was appointed Researcher in Electric Power Systems at the University of Bologna where he was with the Power Systems Laboratory until 2011. In 2010, he received Associate Professor eligibility from the Politecnico di Milano, Italy. Currently, he is Associate Professor at the Swiss Federal Institute of Technology, Lausanne, Switzerland, where he accepted the EOS Holding Chair of Distributed Electrical Systems Laboratory. $\mathrm{He}$ is secretary and member of several IEEE and Cigré Working Groups. He was cochairperson of the technical committee of the ninth edition of the International Conference of Power Systems Transients. His research interests are in the area of smart grids, with particular reference to real-time monitoring and operation, power system protections, power systems dynamics, and power system transients with particular reference to LEMP-interaction with electrical networks.

Rachid Cherkaoui (M'05-SM'07) received both the M.Sc. and Ph.D degrees in electrical engineering in 1983 and 1992, respectively, from École Polytechnique Fédérale de Lausanne (EPFL), Switzerland. Since 1992, he has been senior scientist at EPFL. His research and teaching interests are in electricity market deregulation, in distributed generation and storage and in power system vulnerability. He is senior member of IEEE, member of technical program committees of various conferences, member of CIGRE task forces C5-2, and former IEEE Swiss Chapter officer (20052011). He serves regularly as reviewer for different journals and conferences. Dr. Cherkaoui is the author or co-author of more than 80 scientific publications.

Alberto Borghetti (M'97-SM'03) was born in Cesena, Italy, in 1967. He received the laurea degree (with honors) in electrical engineering from the Universityof Bologna, Italy, in 1992.

Since then, he has been working with the power system group at the University of Bologna, initially as a researcher and since 2004 as Associate Professor of Electric Power Systems. His main research interests concern power system analysis and optimization, power system restoration after blackout, electromagnetic transients due to lightning, distribution system operation, and microgrids. $\mathrm{He}$ is Associate Editor of IEEE TRANSACTIONS ON SMART GRID.

Fabio Napolitano was born in Lucera, Italy, in 1975. He received the M.Sc. degree (with honors) in electrical engineering, in 2003, and the Ph.D degree from the University of Bologna, Bologna, Italy, in 2009.

$\mathrm{He}$ is currently in the Power System Group, Department of Electrical Engineering, University of Bologna. His research interests include power system transients, lightning electromagnetic pulse interaction with electrical networks.

Carlo Alberto Nucci (M'91-SM'02-F'07) is full professor and head of the Power Systems Laboratory of the Department of Electrical Engineering of the Faculty of Engineering of Bologna, where he is now serving as Deputy Dean. Prof. Nucci's research interests cover Power systems transients, with particular reference to those originated by lightning; Dynamics of electric power plants and of power systems, with particular reference to power system restoration after blackouts and to the influence of load modeling on voltage collapse simulations; Distribution Networks/Smart Grid management in the presence of small scale generating plants; Fault location in distribution networks; Phasor Measurement Units for Distribution Networks; Characterization of innovative batteries for electrical vehicles applications; Power Systems Capacitors (medium and low voltage); Waste treatment based on Joule-effect in-situ verification. He is author or coauthor of over 230 scientific papers published on peer-reviewed journals or on proceedings of international conferences, of five book chapters edited by IEE (two), Kluwer, Rumanian Academy of Science and WIT press and of a couple of IEEE Standards and some CIGRE technical brochures. He is a Fellow of the IEEE and of the IET and has received some best paper/technical international awards, including the CIGRE Technical Committee Award. Since January 2006 he is serving as Chairman of Cigré Study Committee C4 'System Technical Performance'. Since January 2012 he is serving as Editor in Chief of the Electric Power Systems Research journal, Elsevier. Prof. Nucci is doctor honoris causa of the University Politehnica of Bucharest and member of the Bologna Science Academy. 